\title{
Semantic Mapping of Discourse and Activity, Using Habermas's Theory of Communicative Action to Analyze Process
}

Fionn Murtagh (1, 2), Monica Pianosi (3), Richard Bull (3)

(1) Department of Computing, Goldsmiths University of London, New Cross, London SE14 6NW, UK

(2) Department of Computing and Mathematics, University of Derby, Kedleston Old Road, Derby DE22 1GB, UK

Email: fmurtagh@acm.org

(3) Institute of Energy \& Sustainable Development

De Montfort University, Leicester LE1 9BH, UK

June 5, 2015

Forthcoming in Quality and Quantity.

The final publication will be available at Springer via

http://dx.doi.org/10.1007/s11135-015-0228-7

"Online First" on SpringerLink:

http://link.springer.com/article/10.1007/s11135-015-0228-7

\begin{abstract}
Our primary objective is evaluation of quality of process. This is addressed through semantic mapping of process. We note how this is complementary to the primacy of output results or products. We use goal-oriented discourse as a case study. We draw benefit from how social and political theorist, Jürgen Habermas, uses what was termed "communicative action". An orientation in Habermas's work, that we use, is analysis of communication or discourse. For this, we take Twitter social media. In our case study, we map the discourse semantically, using the Correspondence Analysis platform for such latent semantic analysis. This permits qualitative and quantitative analytics. Our case study is a set of eight carefully planned Twitter campaigns relating to environmental issues. The aim of these campaigns was to increase environmental awareness and behaviour. Each campaign was launched by an initiating tweet. Using the data gathered in these Twitter campaigns, we sought to map them, and hence to track the flow of the Twitter discourse. This mapping was achieved through semantic embedding. The semantic distance
\end{abstract}


between an initiating act and the aggregate semantic outcome is used as

a measure of process effectiveness.

Keywords: Correspondence Analysis, semantics, multivariate data analysis, text analysis, visualization, social media

\section{Introduction}

\subsection{Analyzing Process: An Innovation Compared to Pat- tern Matching}

Current approaches to analyzing human and social discourse and activity are typically focused on the outputs and results. This is fully justified, when such outputs and results are the main interest, such as for political elections (Bakliwal et al., 2013), or for marketing purposes. In some cases though, the discourse and activity processes, as they evolve, are of major interest in their own right. In Murtagh and Ganz (2015), examples are provided of the tracking of feelings and emotions. Understanding such evolution can be complementary to the outcomes and results.

In this work, we were motivated by social science theorist, Jürgen Habermas, for whom democratic political engagement is a particularly important aspect of socio-political decision-making and action. It will be described in later sections (in particular, subsection 2.4) just how engagement is of particular interest in some areas of social media activity. One example of this is when the impact of social media is, simply, having many users engaged. We move beyond such a trivial form of user engagement. We seek to map out the content of user engagement. Otherwise expressed, this is the process involved in user engagement. This encompasses differing forms of engagement, and strengthening or transformation over time of engagement.

A case study is used in this work. As part of a study for participatory engagement in a social issue, related to environmental citizenship, it is a good example of how we can map out the targeted activity, and the process that is associated with that. From the perspective of this case study, what is sought is substantive engagement by a community, using Twitter.

\subsection{Our Data and Methodology}

This work is concerned with pattern recognition in discourse, involving multiple actors. The trends and patterns in the discourse are the narratives underlying this social interaction. We develop our new approach using a case study. We map out the underlying semantics, in a way that facilitates interpretation of trends and patterns in the discourse.

We use discourse associated with a carefully designed experimental study of social change. The social change we are targeting is in relation to behavioural patterns that are both socially conscious and socially informed. The ultimate aim of our work is how to effect social change. Another starting point in this 
work is the strong belief in democratic, inclusive social influence leading to social change. The theory of communicative action that has been developed by social theorist Jürgen Habermas provides a framework for such social influence leading to social change. In order to have a well designed experimental study, in this article we curtail such communicative action just to Twitter. We set up "campaigns" using Twitter micro-blogging. Then we develop a way to map out, or to track, the effect. From our carefully designed case study, our data collection, and our novel approach to data analysis, we address the following issue: how to understand, and then how to map out, effectiveness "in the small" of such social communicative action. (We may consider effectiveness "in the large" as economic, or political, or other social impact, and consequent change.)

Our novel data analysis is based on Twitter micro-blogging. Notwithstanding very limited, and otherwise tricky, textual data, we will show how we can map the semantics. Let us discuss these general issues somewhat more before we discuss (in section 2) how we use Habermas's theory of communicative action, and then (in section 3) this case-study of Twitter-based narrative.

While counts of Twitter hashtags represent an established way to quantify trends, this does not consider the underlying semantics. Instead we use all relationships in the Twitter data in order to consider the semantics. We map out the tweets and the words used. In this sense, qualitative aspects of the Twitter discourse are included in our methodology. While the basis of our work rests in word use and word counts in each tweet, we take cognizance fully of repeated terms, including hashtags. The qualitative and the quantitative aspects of our data are taken into account. We embed the semantics into a metric space, which is termed a factor space, or a latent semantic space.

A practical problem that we address here is the small amount of text in each tweet. This is a problem to be faced when relying on shared use of terms between tweets. In spite of this, as we will show through our work here, our methodology works very well. We seek to use the given data, perform pattern recognition on it, and not use external lexicons (as in, e.g., Hutto and Gilbert, 2014).

A few further points in regard to our methodology are as follows. Data preprocessing is a crucial part of our analysis chain. Our work can be characterized as unsupervised pattern recognition, and hence data mining or knowledge discovery in data. We will also show how various metrics (in the sense of measurement, not in the mathematical sense of a metric induced by a norm) can be defined. We combine both qualitative (semantic) and quantitative (word count and word usage) information. To advance our methodological objectives in this work, we once again emphasize the essential element of experimental design. 


\section{Semantics of Communication and Discourse, with Orientation and Trend}

\subsection{Defining Effectiveness in Discourse, Arising Out of Habermas's Theory of Communicative Action}

In this work we will develop a new approach to effectiveness assessment for use in communication and discourse, incorporating activity and action. One motivation for our use of Habermas's work is this aspect, that action and activity are taken as integral to communication and discourse. These are all part and parcel of what we observe and analyze.

Our case study is related to Twitter. Such a social medium can be considered as a platform or framework, and in terms of its content, communication is central. For this reason, we begin with a short overview of relevant aspects of the socio-political theorization work of Jürgen Habermas. What we obtain from Habermas's work is twofold, as follows.

1. The overall discourse is comprised of sequences of communicative acts. These are the elements of discourse. Examples: dialogue expressions or other forms of interaction and intervention; tweets or other forms of social media postings. The communicative acts are usually ordered and this can be total order (in the mathematical definition) as befits a chronological order, or a partial order (in the mathematical definition) as befits a hierarchical branching process, i.e. a tree-structure.

2. Habermas's work, as we will next discuss, does not start with the goals of the discourse, followed by what is to be done to achieve those goals. Rather, Habermas's work is based on the study of processes of discourses, with the highlighting of how to get to the endpoint of the goals in efficient and effective ways. It follows for us that effectiveness is to be specified and measured within the discourse process itself.

\subsection{A Short Overview of Habermas's Normative (Hence Process-Oriented) Theory of Communication}

In his major work, The Theory of Communicative Action, Habermas laid out what were termed the normative presuppositions of communicative rationality. Habermas's goal was to set out what such communicative rationality entailed. His motivation for this was to establish rules of behaviour that are involved in discourse. In particular this is discourse of societal importance: public discourse and debate, public engagement in such discourse, and what that implies for citizenship against the big background canvas of democracy.

Communicative rationality includes issues such as the following: presuppositions for discourse; ideal frameworks for debate and discussion; true consensus. A fundamental aspect of communicative rationality is fairness. Involved in this 
are the following: (1) palette or toolset of constitutive contributions to the discourse (such as contributions that are indicative; being recommendations; being evidence based; or being factual declarations, and in the latter case, being ruled by classical logic); (2) inclusivity of representation of those involved in discourse; and (3) balance of soft and hard regulative content of the discourse (through, e.g., requesting, contradicting, permitting, banning, promising, ownership and responsibility). A metalevel of characterization of discourse is on the basis of the discourse being understandable, being reasonable, and being fit for purpose.

On the basis of such a theory of communicative action, truth and correctness, and reasonableness, could all be taken into consideration. Habermas's work was "normative" in the sense that the finality of consensus and engagement come out of the study of the narrative involved in the discourse. This is rather than the "positive" alternative (to "normative" theory) that would start from the desired ideal state, followed by how to achieve that ideal state.

So, for Habermas, it is not goal optimization that is addressed but rather the ideal presuppositions and conditions for discourse that is smoothly running and convergent, and in such a way, being rational and right.

What we find very useful in Habermas's work is that action, that is based on communication, can be judged internally within the context of that overall discourse. Habermas studies human interaction in its "communicative" form, as a smoothly running process, rather than as purely "strategic", hence goaldriven. (See Matušík, 2014).

\subsection{Evaluation of Influence, Effectiveness and Reach in Social Media}

The Twitter campaigns set up and used by us in Pianosi et al. (2013) (which we use in this work) are clearly discourse-based media, and as such they have links with public engagement centred on communicative theory. In order to define, and then measure, terms like "influence", "impact" and "reach", in Pianosi et al. (2013) the following approaches were pursued. Such measures were taken as simply the number of friends, followers, re-tweets, or "like" scores, in a social media (Twitter, Facebook) setting. The practical challenge was discussed of how online activity can actually be measured. Semantics of the online discourse were not included, apart from doing so manually in some cases.

\subsection{Current Top-Down Approaches to Behaviour Change Are Inadequate}

This work of Pianosi et al. (2013) reflects on the strong parallels that social media communication has with public engagement theory. This is based on Habermasian ideals of deliberation, participation, and risk-communication. Top-down information provision, for example relating to the environment, is mere information provision, and is inadequate for behaviour change. Yet top-down communication campaigns both predominate, and are advised, by those involved in social marketing (Collins et al., 2013). However, this rarely manifests itself through 
measurable behaviour change (Lorenzoni et al., 2007). As a result, current research in this field is rife with debate about attitudes and behaviours (Shove, 2010), the value-action gap (Blake, 1999), the importance of habits (Verplanken et al., 2008), and context (Bull et al., 2010), the need for a deliberative turn (Bull et al., 2008), and targeted social marketing (Futerra, 2014).

\subsection{Current Approaches to Measuring Social Media Im- pact}

Social media monitoring was originally adopted by public relations and advertising agencies, who used it as a means to identify negative comments posted on the web about their clients (Barker et al., 2012). It is defined as the activity of observing and tracking content on the social web. Each activity on social media has an outcome, or effect, which can be measured by observing, and then quantifying, specific behaviours. Effects can be one of the following: re-tweets, mentions, favourites, follows, likes, shares, comments, sentiment.

Social media are used by companies and public relations agencies, by local and central governments, who all seek to evaluate the use of social media channels as a communication or engagement tool. In the two cases, evaluation of online success of museums (Finnis et al., 2011), and the Social Media Metric for Federal Agencies (Howto.gov, 2013), the following holds. The emphasis is not on evaluating social media efforts for marketing purposes. Rather, it is to provide organisations with tools to be able to understand if their efforts in engaging citizens have been successful and, crucially, what defines success. It is specifically the emphasis on engagement and collaboration with citizens that makes these approaches different from the marketing strategies. Marketing strategies are more focused on connecting companies with their clients.

\subsection{From Direct Analysis of Activity to Effectiveness and Influence}

As a standard approach, we may consider Bakliwal et al. (2013), relating to Twitter debate in a national election in Ireland. External criteria, through use of external lexicons, were used to characterize tweets in terms of sentiment, in particular positive or negative. In our work here, we are interested in the flow of the tweet signals. In other words, we are primarily interested in the process of that flow, rather than specific, targeted outcomes.

In line with the approaches discussed in the previous subsection, in Pianosi et al. (2013) tools are used that are freely available online to analyse social media traffic. The most basic form of effectiveness thus becomes creating social media conversation. This includes attracting more and new people, engaging them in different actions, and assessing how they participate in conversations, both theme-wise and among themselves. Four main measurement approaches were used: (1) growth of community, (2) engagement (rejoinders, re-tweets, etc.), (3) content indicators, and (4) conversations (number, length, etc.). For each 
category different metrics were defined and compared, using, as we have noted, publicly available software tools.

Quantitative social media (as Twitter here) measurement includes content indicators - e.g., most engaging content, top topics; and analysis of conversations created - length, number of people interacting, topic discussed.

In Pianosi et al. (2013), it is concluded: “... although useful in understanding the effectiveness of a communication campaign in its numerical terms, the proposed methodology can only be the first step of a more in-depth investigation about what people can learn during their online participation, and what is the perceived impact of the process on them, behaviour- or citizenship-wise. Consequently a more in-depth analysis of the characteristic of the community and a content analysis of online conversations is necessary...". We address this, in this work.

\subsection{Seeking New Perspectives on Assessing Effectiveness}

Our aims are thus the following. We seek to analyse the semantics of the discourse in a data-driven way. Since in Pianosi et al. (2013): "top-down communication campaigns both predominate and are advised by those involved in "social marketing" ... . However, this rarely manifests itself through measurable behaviour change ...". Thus our approach is, in its point of departure and vantage point, bottom-up. I.e. our approach is based on the observable data.

Mediated by the latent semantic mapping of the discourse, we will develop semantic distance measures between deliberative actions and the aggregate social effect. We let the data speak (a Benzécri quotation, noted in Blasius and Greenacre, 2014), in regard to influence, impact and reach.

\section{Preliminaries to Measuring Effectiveness: In- put Data Structure, Mapping into a Semantic Space}

\subsection{Sourcing Data on Social Communication from Twitter- Based Social Media}

The general approach to analysis of Twitter conversations taken in Pearce et al. (2014) is based on hashtags (terms preceded with the character "\#" which can be cross-referenced in Twitter) and users (preceded with the "@" character). More than 10 connections were required between users (a connection of a user to another being the explicit use of each other's "@” names). A graph of such exchanges was used for community analysis. The latter, in the case of Pearce et al. (2014), was primarily aimed at the pro and contra viewpoints relative to climate change. Based on such polarization of views, it was found that there was greater prevalence of tweeting in the unsupportive-to-supportive direction (relative to action to counteract climate change). It was nonetheless concluded 
that more work was required: "Content analysis of the tweets could be a possible qualitative approach that could shed light on [...] and provide new knowledge about the content of conversational connections discovered ...". In our work in this article, we look at the conversational connections, starting from what is aiming at being an initiating, instigational and influencing tweet.

In Chew et al. (2010), Twitter-based behaviour (relating to the 2009 H1N1 swine flu) was subjected to content analysis. That included analysis of re-tweets, seeking particular words and phrases, and manual labelling for content and sentiment characterization, followed by analysis of that. Such work was carried out by querying the Twitter data. The queries were sophisticated with many boolean connectives ("and", "or", etc.). Rather than a querying, matching and supervised approach such as this, and used in general for sentiment analysis, our work in this article is data-driven and unsupervised. We map out the underlying semantics of our social media data. The text in the latter (the social media data) is the "sensory surface" McKee (1999) of the underlying semantics.

\subsection{Innovation in This Work: How We Address Effective- ness}

For us, Effectiveness will be the semantic distance between the initiating action, and the net aggregate outcome. This can be statistically tested. It can be visualized. Facets and indeed components of such effectiveness can be further visualized and evaluated.

Essential enabling aspects are as follows. (1) The data structure input, comprising characterization of relevant actions, and characterization of the initiating actions. For all these actions, and the initiating actions, we have their context mode, called "campaign" here. That allows both intra- and inter-campaign analyses. (2) Mapping of this characterization data (presence/absence, frequency of occurrence, mode category) into a semantic space that is both qualitatively (through visualization) and quantitatively analyzed. This semantic space is a Euclidean, factor space.

For visualization we use 2-dimensional projection, but for quantitative analysis, we use the full factor space dimensionality, hence with no loss of information.

Appendix B overviews the Correspondence Analysis methodology. Correspondence Analysis is a latent semantic analysis method. It uses inherent row and column weights. It then uses a weighted distance measure, such that both rows and columns are embedded in the same latent semantic or factor space. The rows and the columns are mapped into the factor, with the same scale. Each row in the factor space is located at the centre of gravity of its associated

columns. Identically, each column in the factor space is located at the centre of gravity of its associated rows. 
Table 1: Twitter data used. Column 1 is the sequence number of the tweet. Column 2 is the tweet. Column 3 has the value 1 if the tweet was an initiating one for a new campaign, and otherwise it is 0 . Column 4 has the value 1 to 8 , indicating the campaign.

\begin{tabular}{|llll|}
\hline Seq. no. & Tweet & Init. - yes $/$ no & Campaign $1,2, \ldots, 8$ \\
\hline 1 & Tweet 1 & 1 & 1 \\
$\ldots$ & $\ldots$ & $\ldots$ & $\ldots$ \\
134 & Tweet 134 & 1 & 3 \\
$\ldots$ & $\ldots$ & $\ldots$ & $\ldots$ \\
$\ldots$ & $\ldots$ & $\ldots$ & $\ldots$ \\
985 & Tweet 985 & 0 & 8 \\
\hline
\end{tabular}

\section{The Data Used}

The eight campaigns in late 2012 were as follows, with the date during which the campaign was carried out, and the theme of the campaign.

1. 1 October to 7 October: Climate change: The big picture and the global consequences.

2. 8 October to 14 October: Climate change: The local consequences.

3. 15 October to 22 October: Light and electricity.

4. 23 October to 28 October: Heating systems.

5. 29 October to 4 November: Sustainable Food choices.

6. 5 November to 11 November: Sustainable Travel choices.

7. 12 November to 18 November: Sustainable Water use.

8. 19 November to 25 November: Sustainable Waste.

Table 1 depicts the initial data set derived from the Twitter data spanning the eight campaigns. There are 985 tweets here. Campaigns were as follows in the succession of tweets: 1 to $63 ; 64$ to $133 ; 134$ to $301 ; 302$ to $409 ; 410$ to $555 ; 556$ to $730 ; 731$ to 843 ; and 844 to 985 . The initiating tweets for the eight campaigns are: $3,65,134,303$ and 304 (which were combined - the two tweets taken together as one), 410, 557, 736 and 846. These initiating tweets are listed in full in Appendix A.

In the first stage of the processing, from all tweets a set of 3056 terms was derived. These terms were essentially the full word set obtained from all tweets. See below, in the following subsection, for an exact specification. Each tweet was cross-tabulated with those terms that were present for it. (Storage-wise, each tweet had $1=$ presence, $0=$ absence values for each of the 3056 terms. In 
some cases there were 2 or 3 presences.) In a second stage of the processing, the term set was reduced to 339 sufficiently often used terms. Some tweets thereby became empty. So the number of usable, non-empty tweets dropped from 985 to 968 non-initiating tweets, plus the 8 initiating tweets. (We have already noted that seven of the eight campaigns had one initiating tweet. Campaign 4 had two successive initiating tweets. We joined these two tweets together into a single initiating tweet for campaign 4.)

For the Correspondence Analysis, the latent semantic mapping method used, a very particular input data set was used, depicted in Figure 1. For the analysis, we distinguish between principal rows (tweets that are not initiating ones) and supplementary rows (tweets that are initiating ones); and principal columns (terms used by the tweets) and supplementary columns (categorization in regard to the campaign). See Figure 1. The analysis that embeds rows and columns in a factor space is carried out on the principal rows and columns, i.e. the regular discourse (non-initiating) tweets crossed by the terms that characterize them. Into that factor space, the supplementary rows and columns are projected, i.e. respectively the initiating tweets, and the campaign categories.

The data to be analyzed then was as follows.

- Principal rows: the set of 968 retained tweets, that do not include the initiating tweets.

- Supplementary rows: the set of 8 initiating tweets.

- Principal columns: the set of 339 terms retained.

- Supplementary columns: the set of 8 "indicators" for the 8 campaigns.

\subsection{Preprocessing the Content of the Tweets}

In this and the next subsection, we explain how we select the term set used to characterize each tweet in the overall Twitter discourse.

Only alphabetic characters are retained. So @, \# are dropped but we can generally spot user or hashtag terms from the remaining term stump. Numerical data are dropped including dates, since we will focus exclusively on word-based data. Punctuation and special characters go too, e.g. in URLs. We could handle these were it advisable to do so.

The html expression for ampersand ("\&amp;"), in our processing left with a rump, "amp", is substituted with "and".

All upper case is set to lower case (with no loss of information involved).

We deleted "ll" (left over from e.g. "I'll"), "s" (from e.g. "it's"), and "t" (from e.g. "isn't" or "wouldn't").

We find 3323 terms used in the original set of 985 tweets.

Terms on a stopword list ("and", "the", etc.) are deleted, and this decreases the 3323 term set to 3056 terms. In Murtagh (2005, section 5.3, "Tool words: between analysis of form and analysis of content") we discuss the case 


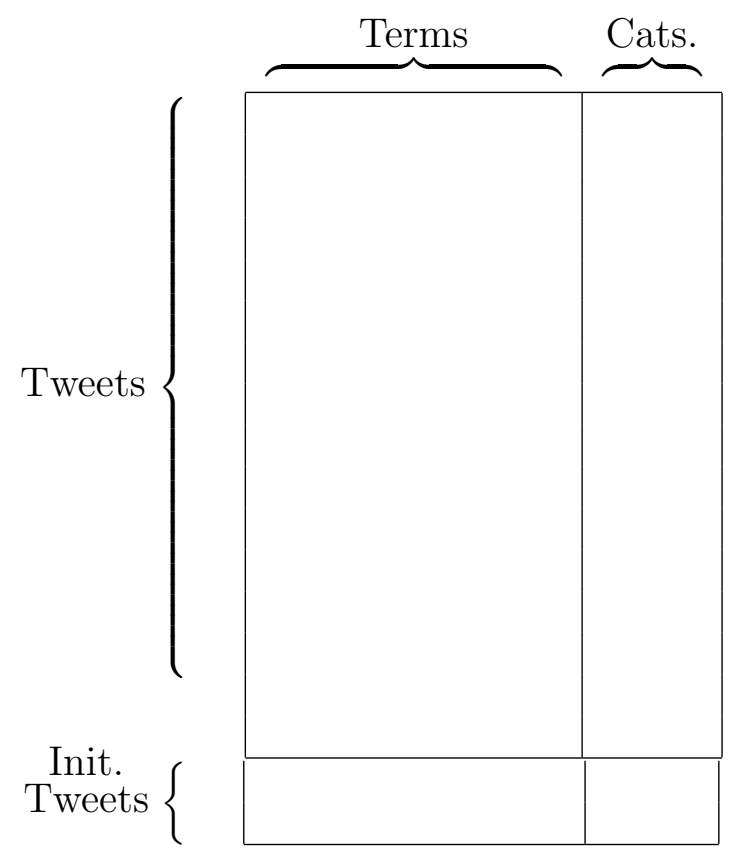

Figure 1: Upper left, Tweets $\times$ Terms: the majority of values are 0 , indicating absence of term in the tweet. Some values are 1 (and a few are 2, or even 3) indicating presence of term in the tweet. Upper right, Tweets $\times$ Categories, where a value of 1 in the relevant campaign column is associated with that particular tweet. Otherwise values are 0 . Lower left, Initiators $\times$ Terms: the 1 and 0 values are as for Tweets $\times$ Terms. Lower right, Initiators $\times$ Categories, i.e. Campaigns: each row has a campaign $=1$ and otherwise 0 . 
for considering such function words or "tool words" in many languages, that are especially important for characterizing style. However in this present work, we are not interested in distinguishing between styles in this way (or emotional content, see Pennebaker, 2011).

\subsection{Preprocessing the Tweets $\times$ Terms Matrix}

The tweets $\times$ terms cross-tabulation is set up, with frequency of occurrence values. The greatest frequency of occurrence value is 3 , and in most cases of presence of a term in the tweet, it is 1 . The cross-tabulation matrix is very sparse, with most values equal to 0 .

In order to facilitate and even to make possible the comparison of all tweets in the Twitter discourse, we require each set of presences of terms over all tweets to be at least 5 , and also that the term be present in 5 tweets. Very rarely used terms would hinder our analysis, due to their exceptionality. Here, however, we want to focus on semantic non-exceptionality, i.e. semantic commonality. Our thresholds of 5 were such that rarely used terms were pinpointed, and not at the cost of removing too many terms.

The 968 retained (non-initiating) tweets, and the 8 initiating tweets, are crossed by 339 terms.

\section{Data Analysis}

\subsection{Semantic Mapping of Tweets, Terms and Categories through Correspondence Analysis}

Again, note the background description of Correspondence Analysis in Appendix B. Factors, in decreasing order of importance, provide latent semantic components. The supplementary rows and columns are projected into the factor space. The principal rows are the non-initiating tweets. The principal columns are the set of terms used in this discourse. The supplementary rows are the initiating tweets. The supplementary columns are the campaign indicators.

Each term is at the centre of gravity of that term's tweets. Each tweet is at the centre of gravity of that tweet's terms.

The factor space is a semantic space in that it takes account of all interrelationships - between all tweets, between all terms, between all tweets and all terms.

Typically we visualize this semantic, factor representation of the data by taking two factors at a time. Planar projections lend themselves to such display. In the analysis discussion to follow, we tidy up these displays, in order to highlight useful and/or important outcomes. 


\subsection{Semantically Locating the Initiating Tweets and the Net Overall Campaign Tweets}

Our first analysis takes the principal factor plane and shows the following in Figure 2: the 8 tweets that initiated the campaigns, and the net aggregate campaigns, given by the centres of gravity of the 8 campaigns. We projected the supplementary rows, and the supplementary columns, see Figure 1. As we noted above, the actual definition of the factors was from the principal rows, i.e., all tweets save the initiating ones; and the principal columns, i.e., the word set used in the Twitter discourse. The information explained by these factors (the inertia of the factors, noted in Figure 2) is just under 2 percent. While the principal factor plane accounts for a relatively small amount of information in our data, this is typical for sparse input data (here, tweets crossed by terms). The principal factor plane is the mathematically best planar representation, hence summary, of our data. In this plane, of factors 1 and 2, Figure 2 shows the instigating tweet ("tic1", etc.) and the net overall effect ("C1", etc.).

We see that campaigns $3,5,8$ have initiating tweets that are fairly close to the net overall campaign in these cases. By looking at all tweets, and all terms, it is seen that the campaign initiating tweets, and the overall campaign means, are close to the origin, i.e. the global average. That just means that they (respectively, initiating tweets, and means) are relatively unexceptional among all tweets. While the information that we find in our data is very faint, nonetheless we have an excellent visualization of this information, that we will further analyze now.

While we see that tweets initiating campaigns $3,5,8$ are the closest to their respective campaign means, this is based on the best fitting planar, twodimensional projection. It is based on the best factor plane, defined by factors 1 and 2. But the entire semantic space is of dimensionality 338. (This is explained as follows. The principal row set is 968 tweets. The principal column set is 339 tweets. In Correspondence Analysis, the dimensionality of the factor space is less than or equal to $\min (339-1,968-1)$.)

We now look at the distances between tweets initiating campaigns 1 to 8 , relative to their respective campaign means, in the factor space of full dimensionality, 338. We are using all information, providing a different, and more complete, perspective. See Figure 3, that summarizes what we find. We find that campaign 7 shows the most effectiveness by its initiating tweet, followed by campaigns 6 , then 4 , then 5 , then 1 . Increasingly less effective are 3,8 and 2 .

\subsection{Statistical Significance of Effectiveness}

Here we show how we can test the statistical significance of effectiveness.

The campaign 7 case, with the distance between the tweet initiating campaign 7 , and the mean campaign 7 outcome, in the full, 338-dimensional factor space is equal to 3.670904. (This is used in Figure 3.)

Compare that to all pairwise distances of non-initiating tweets. We verified 
8 campaign initiating tweets, and centres of gravity of 8 campaigns

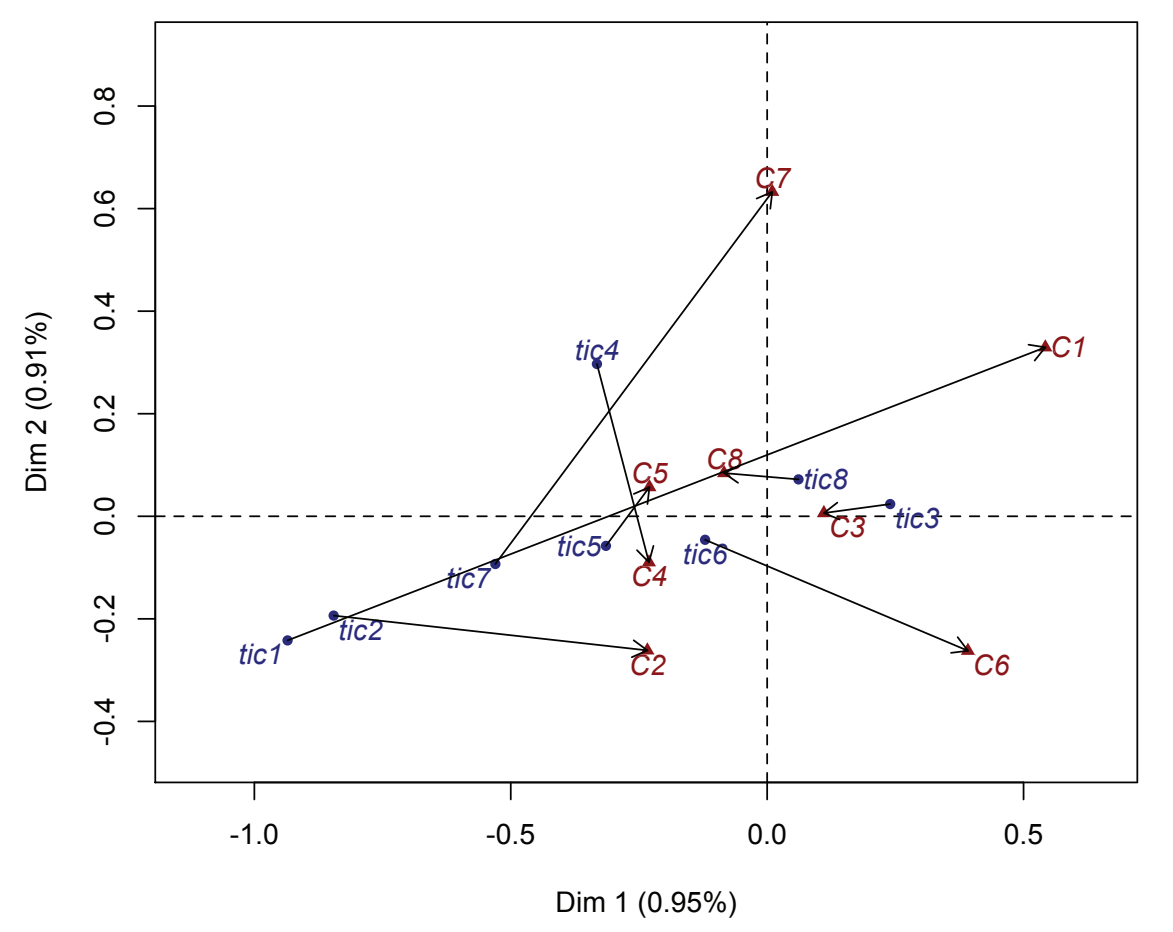

Figure 2: The campaign initiating tweets are labelled "tic1" to "tic8". The centres of gravity of the campaigns, i.e. the net aggregate of the campaigns, are labelled "C1" to "C8". In each case, the tweet initiating the campaign is linked with an arrow to the net aggregate of the campaign. The percentage inertia explained by the factors, "Dim 1" being factor 1, and "Dim 2" being factor 2, is noted. 


\section{Distances between initiating tweet and campaign mean}

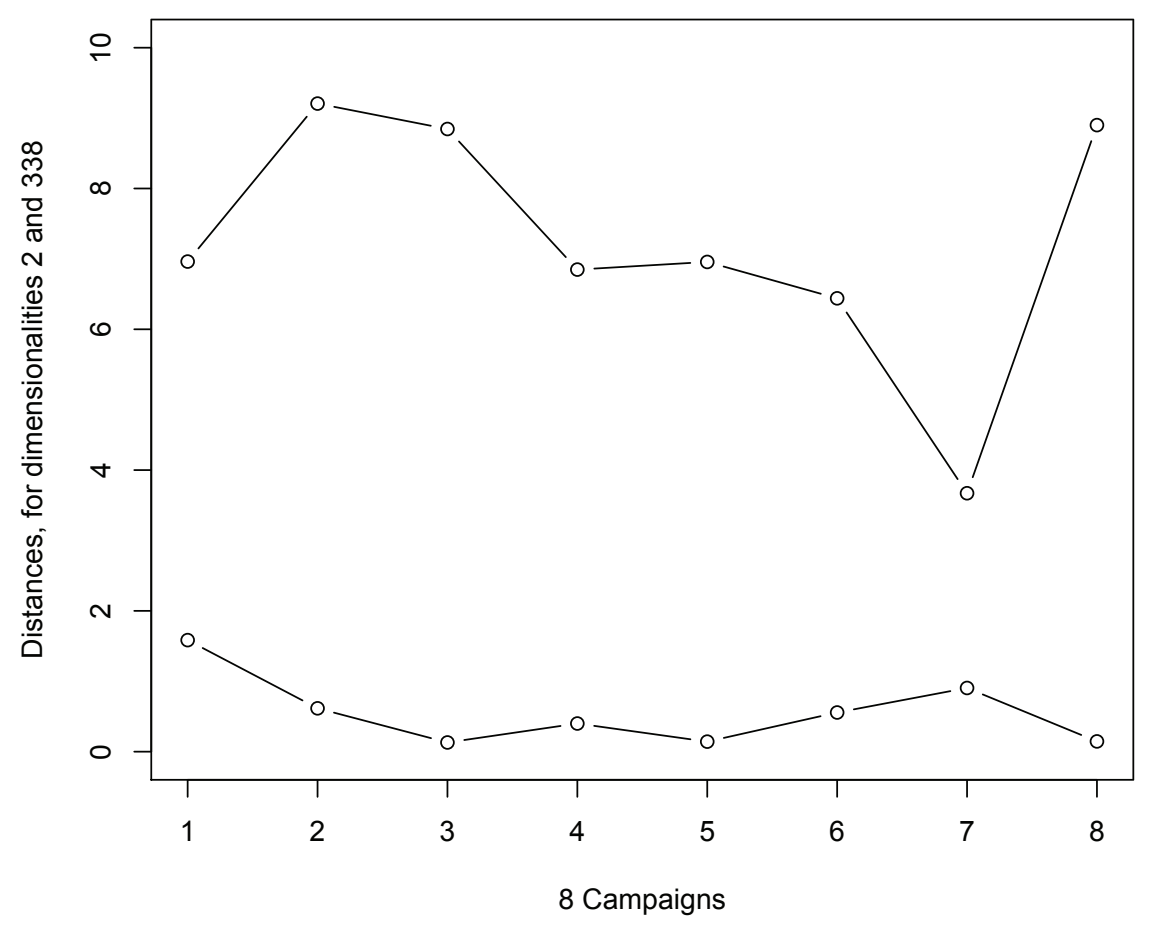

Figure 3: For the 8 campaigns, shown are the Euclidean distances between the campaign initiating tweets and the respective centres of gravity of the campaigns, or net overall campaigns. The lower curve is for the principal factor plane, hence the Euclidean distances between "tic1" and "C1", etc., as shown in Figure 2. The upper curve is for the full semantic, factor space dimensionality. 
that these distances are normally distributed, with a small number of large distances. By the central limit theorem, for very large numbers of such distances, they will be normally distributed. Denote the mean by $\mu$, and the standard deviation by $\sigma$. Mean and standard deviation are defined from distances between all non-initiating tweets, in the full dimensionality semantic (or factor) space. We find $\mu=12.64907, \mu-\sigma=8.508712$, and $\mu-2 \sigma=4.368352$.

We find the distance between initiating tweet and mean outcome, for campaign 7 , in terms of the mean and standard deviation of tweet distances to be: $\mu-2.168451 \sigma$. Therefore for $z=-2.16$, the campaign 7 effectiveness is significant at the $1.5 \%$ level (i.e. $z=-2.16$, in the two-sided case, has $98.5 \%$ of the normal distribution greater than it in value).

In the case of campaigns $1,4,5,6$, their distances between initiating tweet and mean outcome are less than $90 \%$ of all tweet distances. Therefore the effectiveness of these campaigns is in the top $10 \%$ which is not greatly effective.

In the case of campaigns 3 and 8 , we find their distances to be less than $80 \%$ of all tweet distances. So their effectiveness is in the top $20 \%$.

Finally, campaign 2 is the least good fit, relative to initiating tweet and outcome.

\subsection{Detailed Look at Campaign 7}

Having found campaign 7 to be the best from the point of view of proximity of cause and intended effect, we now look in somewhat more detail at this campaign. Campaign 7 was the best campaign in the full semantic dimensionality context, as we have observed.

Campaign 7 relates to Sustainable Water use, cf. Appendix A. Including the initiating tweet, there are 112 tweets (that have not become empty of terms in our term filtering preprocessing) in campaign 7 , and there are 176 terms that appear at least once in the set of tweets. We now use Correspondence Analysis on just this campaign 7 data.

We show the factors 1, 2 plane with the tweets, noting where the initiating tweet is located in this projection, see Figure 4; and then we show the most important terms, see Figure 5. In the latter, note the locations of tweeter names, @TheActualMattyC, @TheEAUC, @BeverleyLad.

Interestingly, in Figure 5, relating to water, the relevant terms for this campaign are projected near to the origin. That they completely dominate the semantics in this way is most reassuring, in regard to our methodology. We found that the following terms, in most cases used in the Twitter discourse with hashtags, were all projected very close to the origin, and appear bunched in Figure 5: "bottledwater", "tap", "tapwater", "sustainablewater", "sustainable", "sustainability", "waterfootprint".

The remaining story narrated by the principal plane view of campaign 7 is very largely a three-way interplay of tweeter personalities, @TheActualMattyC, @TheEAUC, @BeverleyLad. Note how they are reduced in our preprocessing (cf. Figure 5) to, respectively, "theactualmattyc", "theeauc" and "beverleylad". Respectively these are associated with: positive F1, positive F2; negative F1, 
Campaign 7: Factors 1, 2, with 10 most contributing tweets

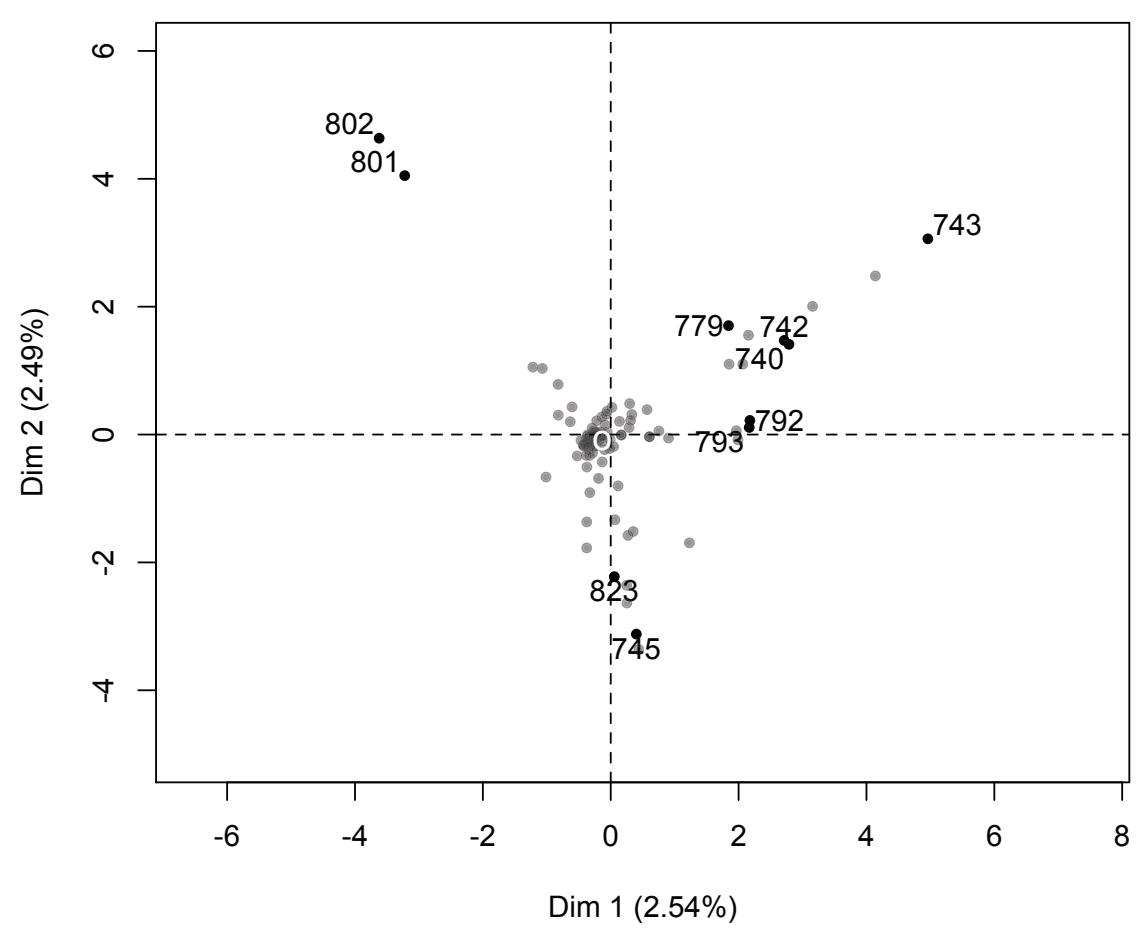

Initiating tweet: white "o". Otherwise sequence numbers of tweets in this campaign.

Figure 4: Principal factor plane for campaign 7. Just the tweet set for this campaign is used, including the initiating tweet. Terms are used that appear at least once in the set of tweets. The input data used is 112 tweets crossed by 176 terms. The 10 most contributing tweets are labelled here (with their tweet sequence numbers), and the initiating tweet (the white "o" just to the lower left of the origin) is also displayed. The positions of all other tweets are shown as gray points. (These are particularly numerous around the origin.) 
Campaign 7: Factors 1, 2, with 15 highest coordinate terms

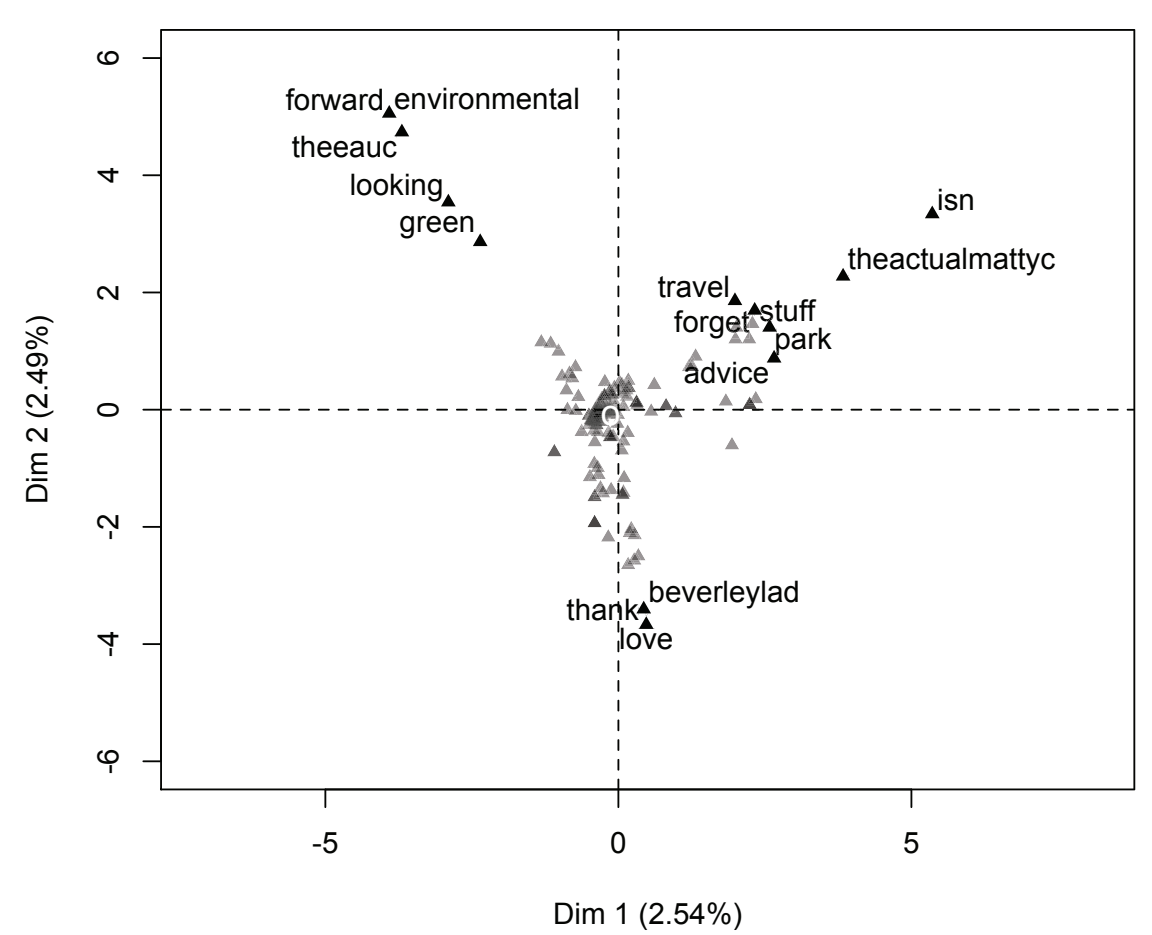

Figure 5: The same data is used as in Figure 4. The 15 highest coordinate values of terms are labelled here. 
positive F2; and relatively neutral F1, negative F2 (where F1 and F2 are factor 1 and factor 2 coordinates). Regarding the last of these tweeter individuals, the term "love" appears in a tweet indicating "we'd love a cycling Leicester", and the word "thank" appears quite a few times. (The word "thanks" is used a lot also, and is projected just a little less highly in terms of negative F2 loading, compared to "thank".) In Figure 5, projections of "beverlylad" and "thank" are superimposed, and projections of "forward" and "environmental" are also superimposed.

To conclude on this look at campaign 7, we have noted that all terms related to water are around the origin. Such terms define the origin as the dominating, or average, theme. That fundamental aspect confirms the overall focus of the tweets in campaign 7 on water. Above and beyond that fundamental aspect, we find a three-way interplay of tweeter personalities, @TheActualMattyC, @TheEAUC, and @BeverleyLad.

\subsection{Scalability of This Method for Measuring Effective- ness}

Consider the scaling up of our approach to vast numbers of tweets. Our approach is highly scalable. We do not in fact need to determine the factors, with their projections and contributions (see Appendix B, for background on Correspondence Analysis). Instead we can directly use the $\chi^{2}$ metric between an initiating (or, more generally, an instigating) tweet, and the aggregated average. Consider, for now, the overall average, i.e. the origin in the factor space.

Denote the tweets by terms matrix in Figure 1 as consisting of values $k_{i j}$. Denote the matrix as $k_{I J}$ for $i \in I, j \in J$. The frequency table is formed from this as $f_{i j}=k_{i j} / k$ where $k=\sum_{i, j} k_{i j}$. The distribution function of the tweets is the marginal set of frequencies, $f_{I}=\left\{f_{i}: i \in I\right\}$ where $f_{i}=\sum_{j} f_{i j}$. What we analyze are the profiles, in both the cloud of tweets, and the cloud of terms. A tweet profile is $f_{i j} / f_{i} \forall j \in J$. A succinct notation for this is that the $i$ th tweet profile is $f_{J}^{i}$.

Next, we come to the origin (centre of gravity) of the axes (i.e. the factors) established by the Correspondence Analysis. For the cloud of tweets, it is $f_{J}$. The centre of the cloud of words is, analogously, $f_{I}$. The distance squared of profile $i$ to the centre of the cloud of tweets is then given by: $\left\|f_{J}^{i}-f_{J}\right\|_{f_{J}}^{2}$. This is a weighted Euclidean distance squared. It is termed the $\chi^{2}$ distance, of centre $f_{J}$. It is identical to the Euclidean distance squared of tweet $i$, relative to the origin, in the Correspondence Analysis factor space (i.e. each tweet and each word is defined by their factor coordinates, or projections). The $\chi^{2}$ distance squared is defined thus:

$$
\left\|f_{J}^{i}-f_{J}\right\|_{f_{j}}^{2}=\sum_{j} \frac{1}{f_{j}}\left(\frac{f_{i j}}{f_{i}}-f_{j}\right)^{2}
$$

All terms here are computationally efficient to calculate. (The $\chi^{2}$ distance is linear in the number of words retained for the analysis, viz. the set $J$.) Therefore 
it is very straightforward to generalize our effectiveness measuring approach to massive data sets.

\subsection{Scalability of Tracking and Mapping Process}

An aspect of our work is the importance of the aggregate, or mean, profile. In Murtagh and Contreras (2015), it is shown how data piling or concentration is typical of very high dimensional data. In Murtagh and Contreras (2015) it is reported on how data become very concentrated in high dimensions. Dimensions of up to one million are studied. This is indicative to the scalability of our work, certainly in regard to very high ambient dimensions.

Further results on analysis of Twitter data, using five 1000-tweet sequences from newspapers in the US, the UK, Ireland, France and Germany, are at issue in Murtagh and Ganz (2015). In that work, the focus is on pattern finding, followed by semantic centrality of such patterns.

\section{Conclusions}

We have developed a new approach to assessing effectiveness, based on the process of discourse. A causal element is used, and this is compared to the overall aggregate of the course of the discourse.

We have studied this comparatively, using 8 different "campaigns". We have traced out the semantic path from initiating tweet to the mean tweet of the associated campaign. We noted the differences between campaigns. First, we did this using the most salient two-dimensional latent semantic, or factor, subspace. That was useful for display purposes. For further analysis, we used the full dimensionality space, using all available information and avoiding any approximation.

We noted differences in the campaign processes, by semantically tracking them. For example, campaign 3 overall was closest to its initiating tweet in the two-factor projection. But with all information in use, campaign 7 was the most effective campaign of all, in the sense of the initiating tweet being closest to the overall semantic mean of that campaign. We have also developed a statistical test of significance of effectiveness.

Planar projections in our semantic, factor space allow further analysis of outcomes, backed up by visualization. We looked in detail at campaign 7 . We checked up on the the most influential tweets, and the most revealing terms associated with the underlying (latent semantic) components. In some cases, this indicated who (the tweeter, @) or what themes (hashtag, \#) were dominant. In other cases particular words were at issue.

Our word set used was a carefully selected one. Nonetheless it was flexibly open to various grammatical forms, and to stumps of words serving as proxies for words containing punctuation, web addresses, or other non-standard character strings. We avoided punctuation (including multiple exclamation marks) and special characters. We treated words that were run together as a composite 
word (possibly later removed in our preprocessing as a rare word). We avoided numeric data as being non-interpretable for the type of narrative that is of interest to us here. We allowed URLs or abbreviations to be retained in our analysis as stumps of words (again possibly removed due to rarity).

We noted in our introduction how top-down, command or managerially imposed approaches to behaviour change have been found to be often inadequate and ineffective. Our motivation was to accept a Habermasian view that democratic, inclusive engagement through communicative processes is a better way to bring about behaviour change. Our approach to quantifying effectiveness is in this context of being process-based and data-driven.

\section{References}

[1] Barker, M., Barker, D.I., Bormann, N.F., Neher, K.E.: Social Media Marketing. A Strategic Approach. Andover UK, Cengage Learning (2012)

[2] Bakliwal, A., Foster, J., van der Puil, J., O’Brien, R., Tounsi, L., Hughes, M.: Sentiment analysis of political tweets: towards an accurate classifier, Proceedings of the Workshop on Language in Social Media, LASM 2013, Association for Computational Linguistics, pp. 49-58 (2013)

[3] Benzécri, J.-P.: L'Analyse des Données, Tome I Taxinomie, Tome II Correspondances, 2nd ed. Paris, Dunod (1979)

[4] Benzécri, J.-P.: Correspondence Analysis Handbook. Basel, Dekker (1994)

[5] Blake, J.: Overcoming the "Value-Action Gap" in environmental policy: tensions between national policy and local experience. Local Environment. $4(3), 257-278$ (1999)

[6] Blasius, J., Greenacre, M. (Eds.): Visualization and Verbalization of Data. Boca Raton, FL, Chapman \& Hall/CRC Press (2014)

[7] Bull, R., Petts, J., Evans, J.: Social learning from public engagement: dreaming the impossible? Journal of Environmental Planning and Management, 51(5), 701-716 (2008)

[8] Bull, R., Petts, J., Evans, J.: The importance of context for effective public engagement: learning from the governance of waste. Journal of Environmental Planning and Management, 8(53), 991-1009 (2010)

[9] Chew, C., Eysenbach, G.: Pandemics in the age of Twitter: content analysis of tweets during the 2009 H1N1 outbreak. PLoS ONE, 5(11), e14118, 13 pp. (2010)

[10] Collins, J., Thomas, G., Willis, R., Wilsdon, J.: Carrots, sticks and sermons: influencing public behaviour for environmental goals. A Demos/Green Alliance report produced for DEFRA, Demos/Green Alliance, 
55 pp. (2003) (www.demos.co.uk/files/CarrotsSticksSermons.pdf retrieved 13 April 2014)

[11] Finnis, J., Chan, S., Clements, R.: Let's get real. How to Evaluate Online Success? Report from the Culture24 Action Research Project. Brighton, 40 pp. (2011) (http://www.keepandshare.com/doc/3148918/culture24howtoevaluateonlinesuccess-2-pdf $\quad$-september-19-2011-11-15-am-2-5meg?da=y retrieved 13 April 2014)

[12] Futerra: The Rules of the Game: The Principles of Climate Change Communication, Futerra Sustainability Communications Ltd., London, 5pp. (2005) (www.futerra.co.uk/downloads/RulesOfTheGame.pdf retrieved 13 April 2014)

[13] Howto.gov: Social Media Metrics for Federal Agencies, U.S. General Services Administration (2013) (http://www.howto.gov/social-media/usingsocial-media-in-government/metrics-for-federal-agencies retrieved 13 April 2014)

[14] Hutto, C.J., Gilbert, E.: VADER: A parsimonious rule-based model for sentiment analysis of social media text, Proceedings of the Eighth International Conference on Weblogs and Social Media, ICWSM 2014. Ann Arbor, Michigan, USA, June 1-4 (2014)

[15] Le Roux, B., Rouanet, H.: Geometric Data Analysis: From Correspondence Analysis to Structured Data Analysis. Dordrecht, Kluwer Academic (2004)

[16] Lorenzoni, I., Nicholson-Cole, S., Whitmarsh, L.: Barriers perceived to engaging with climate change among the UK public and their policy implications. Global Environmental Change, 17(3-4), 445-459 (2007)

[17] Matušík, M.B.: Jürgen Habermas, philosophy and social theory http://www.britannica.com/EBchecked/topic/250787/JurgenHabermas/281673 /Philosophy-and-social-theory (retrieved 21 March 2014)

[18] McKee, R.: Story: Substance, Structure, Style, and the Principles of Screenwriting. London UK, Methuen (1999)

[19] Murtagh, F.: Correspondence Analysis and Data Coding with R and Java. Boca Raton, FL, Chapman \& Hall/CRC (2005)

[20] Murtagh, F., Ganz, A.: Pattern recognition in narrative: Tracking emotional expression in context, preprint, http://arxiv.org/abs/1405.3539 (2015)

[21] Murtagh, F., Contreras, P.: Big data scaling through metric mapping: Exploiting the remarkable simplicity of very high dimensional spaces using Correspondence Analysis, in preparation (2015). 
[22] Pearce, W., Holmberg, K., Hellsten, I., Nerlich, B.: Climate change on Twitter: topics, communities and conversations about the 2013 IPCC Working Group 1 report, PLoS ONE, 9 (4), 11 pp., e94785 (2014)

[23] Pennebaker, J.W.: The Secret Life of Pronouns: What Our Words Say About Us. New York, Bloomsbury Press (2011)

[24] Pianosi, M., Bull, R., Rieser, M.: Impact, influence and reach: Lessons in measuring the impact of social media, pp. 36, preprint (2013)

[25] Séguéla, J., Saporta, G.: A comparison between latent semantic analysis and correspondence analysis, presentation, CARME, Correspondence Analysis and Related Methods Conference, Rennes, France (2011) http//carme2011.agrocampus-ouest.fr/slides/Seguela_Saporta.pdf

[26] Shove, E.: Beyond the ABC: climate change policy and theories of social change. Environment and Planning. 42, 1273-1285 (2010)

[27] Verplanken, B., Walker, I., Daves, A., Jurasek, M.: Context change and travel mode choice: Combining the habit discontinuity and self-activation hypotheses. Journal of Environmental Planning and Management. 53(8), 991-1009 (2008)

\section{Appendix A: Our 8 Campaign Initiating Tweets}

The following are the campaign initiating tweets, in full. For campaign 4, the two initiating tweets were merged together. DMU stands for De Montfort University.

Campaign 1: Introducing \#climatechange! Is the climate changing? What are the observed changes?Are humans causing it? Discuss http://t.co/cMUOmbEt \#dmuCC

Campaign 2: Do you feel \#climatechange is a distant issue? Read and listen to the climate witnesses in the UK http://t.co/FLWaTqTb

Campaign 3: Goodmorning \#DMU!! How was your weekend? Did you participate in the \#marathon? We are talking about electricity this week! \#dmuelectricity

Campaign 4:Goodmorning \#DMU!! How was your weekend? We are talking about gas and heating this week! \#dmuenergy Wishing you all a nice \#ecomonday!

Campaign 4: Connect with us to discover what \#DMU is already doing to cut its \#gas use and tell us what you think we could all do to make it better!

Campaign 5: Goodmorning \#DMU!! We talk about \#sustainable food this week. We have a question for you! What do you think does Sustainable Food mean? 
Campaign 6: Here I am, fueled with caffeine! This week we will be talking in particular of \#transport. How do you get from home to \#DMU? \#dmutransport

Campaign 7: New post! \#Sustainable \#Water - Are you familiar with the concept of \#WaterSecurity? http://t.co/T9QYVlTJ \#DMU \#climate \#sustainabledmu

Campaign 8: @SustainableDMU \#MeatFreeMonday seems to have latched itself into my brain! Not a big meat eater but like having a dedicated veggie day!

As discussed in subsections 4.1, 4.2, a set of 339 terms was ultimately selected as the set of all employable words used in the discourse. The terms retained for these particular initiating tweets, with frequency of occurrence, are as follows. For campaigns 1 through 8, we see that we have, respectively, summed frequencies of occurrence of terms: 4,4,7,14,10,6,7,5.

Campaign 1: climate climatechange dmucc http [1 occurrence each]

Campaign 2: climate climatechange http read [1 occurrence each]

Campaign 3: dmu electricity goodmorning participate talking week weekend [1 occurrence each]

Campaign 4: cut dmu dmuenergy ecomonday gas goodmorning heating nice talking tell week weekend [dmu, gas: 2 occurrences; otherwise 1 occurrence]

Campaign 5: dmu food goodmorning mean question sustainable talk week [food, sustainable: 2 occurrences; otherwise 1 occurrence]

Campaign 6: dmu dmutransport home talking transport week [1 occurrence each]

Campaign 7: climate dmu http post sustainable sustainabledmu water [1 occurrence each]

Campaign 8: day meat meatfreemonday sustainabledmu veggie [1 occurrence each]

The campaign 4 tweet was a merged one (from original tweets 303, 304). In campaign 4 , the term "gas" is both word and hashtag. It is easy to go back to the original tweets and see the hashtags, or the tweeters. We keep the "http" part of the URL since it informs us that a web address is in the tweet. 


\section{Appendix B: Correspondence Analysis}

Correspondence Analysis provides access to the semantics of information expressed by the data. The way it does this is to define semantically each observation (a tweet here), or row vector, as the average of all attributes (term here) that are related to it. Similarly it defines semantically each attribute, or column vector, as the average of all observations that are related to it.

This semantic mapping analysis is as follows:

1. The starting point is a matrix that cross-tabulates the dependencies, e.g. frequencies of joint occurrence, of an observations crossed by attributes matrix.

2. By endowing the cross-tabulation matrix with the $\chi^{2}$ (chi squared) metric on both observation set (rows) and attribute set (columns), we can map observations and attributes into the same space, endowed with the Euclidean metric.

3. Interpretation is through (i) projections of observations, attributes onto factors; (ii) contributions by observations, attributes to the inertia of the factors; and (iii) correlations of observations, attributes with the factors. The factors are ordered by decreasing importance.

Correspondence Analysis is not unlike Principal Components Analysis in its underlying geometrical bases. While Principal Components Analysis is particularly suitable for quantitative data, Correspondence Analysis is appropriate for the following types of (non-negative valued) input data: frequencies, contingency tables, probabilities, categorical data, and mixed qualitative/categorical data.

The factors are defined by a new orthogonal coordinate system endowed with the Euclidean distance. The factors are determined from the eigenvectors of a positive semi-definite matrix (hence with non-negative eigenvalues). This matrix which is diagonalized (i.e. subjected to singular value decomposition) encapsulates the requirement for the new coordinates to successively best fit the given data.

The "standardizing" inherent in Correspondence Analysis (a consequence of the $\chi^{2}$ distance) treats rows and columns in a symmetric manner. One byproduct is that the row and column projections in the new space may both be plotted on the same output graphic presentations (the principal factor plane given by the factor 1 and factor 2 coordinates; or other pairs of factors).

\section{From Frequencies of Occurrence to Clouds of Pro- files, each Profile with an Associated Mass}

From the initial frequencies data matrix, a set of probability data, $f_{i j}$, is defined by dividing each value by the grand total of all elements in the matrix. In 
Correspondence Analysis, each row (or column) point is considered to have an associated weight. The row weight is the row sum, divided by the overall data matrix total. The column weight is the column sum, divided by the overall data matrix total.

Next row profiles are defined as the row frequencies divided by the row weight (also termed the mass). Similarly we have column profiles. The $\chi^{2}$ distance between profiles is a weighted Euclidean distance. It is an appropriate distance for what are, initially here, categorical data.

We thus look on our row points (our tweets) as a cloud of points endowed with the $\chi^{2}$ distance. Similarly our column points (our words) are a cloud of points that are also endowed with the $\chi^{2}$ distance.

Just like in classical mechanics, we consider the inertia of these clouds. To begin with we have their total inertia, that is the inertia about their centre of gravity. The centre of gravity is the weighted mean. The way that the cloud of row points, and the cloud of column points, have been defined, is that the inertias of these two clouds are identical.

\section{Output: Cloud of Points Endowed with the Eu- clidean Metric in Factor Space}

Decomposing the moment of inertia of the cloud of row points (the cloud of tweets) and the cloud of column points (the cloud of words) furnishes the principal axes of inertia, defined from a singular value decomposition. The inertia about the principal axes is given by the eigenvalues. The principal axes themselves are defined from the eigenvectors. The principal axes are termed factors. Latent variables, or latent semantic axes, are also terms that can be used.

There is the following invariance relationship. The $\chi^{2}$ distance between two rows (two tweets), or between two columns (two columns), is identical to the Euclidean distance between the two rows, or respectively the two columns, in the factor space. The latter, the factor space, allows us to display the data.

The projection of row points and column points on the factors express the information. The total information content of either row set, or column set, is the cloud inertia. Associated with the factors is the information in our data, arranged by decreasing importance. The information importance is measured by inertia about the axes, or factors.

In addition to projections on the factorial axes, in Correspondence Analysis, we also consider the contributions to the inertia, and the correlations (of rows, or of columns, with the factors). 


\section{Analysis of the Dual Spaces, and Supplementary Elements}

The factors in the two spaces, of rows/observations and of columns/attributes, are inherently related. Each row (tweet) coordinate in the factor space is defined by the barycentre (or centre of gravity) of the coordinates of the column (word) coordinates; and vice versa. Not only can we pass from one cloud to the other, but the two clouds (of rows, and of columns) are displayable on the same graphic output. This is because the two clouds that are endowed with the $\chi^{2}$ distance to start with, are projected into (or embedded in) the factor space. The factor space, as noted above, is endowed with the Euclidean distance. The Euclidean distance is particularly appropriate for display or visualization.

Qualitatively different elements (i.e. row or column profiles), or ancillary characterization or descriptive elements may be placed as supplementary elements. This means that they are given zero mass in the analysis, and their projections are determined using the transition formulas. This amounts to carrying out a Correspondence Analysis first, without these elements, and then projecting them into the factor space following the determination of all properties of this space.

\section{In Summary}

Correspondence Analysis is thus the inertial decomposition of the dual clouds

of weighted points. It is a latent semantic decomposition, where the role of the term frequency and inverse document frequency (TF-IDF) weighting scheme is instead through the use of (i) profiles and masses, (ii) with the $\chi^{2}$ distance. See Séguéla and Saporta (2011) for a discussion of both methods, Correspondence Analysis and Latent Semantic Indexing. Further background description on Correspondence Analysis can be found in Benzécri $(1979,1994)$, Le Roux and Rouanet (2004), Murtagh (2005). 